\title{
Divisors of Mersenne Numbers $10,000<p<15,000$
}

\section{By Sidney Kravitz}

Riesel has previously presented [1] a table of the smallest factors, $q$, of the Mersenne numbers, $M_{p}=2^{p}-1, p$, a prime, subject to the conditions that $p<$

Divisors of Mersenne Numbers

$q$ Divides $M_{p}=2^{p}-1 ; K=(q-1) / 2 p$

\begin{tabular}{|c|c|c|c|c|c|c|c|c|c|}
\hline$p$ & $K$ & $p$ & $K$ & $p$ & K & $p$ & $K$ & $p$ & $K$ \\
\hline 10,007 & 12 & 10,883 & 1 & 12,037 & 3 & 12,923 & 1 & 13,841 & 3 \\
\hline 10,067 & 25 & 10,891 & 9 & 12,041 & 75 & 12,941 & 4 & 13,883 & 1 \\
\hline 10,091 & 1 & 10,949 & 7 & 12,049 & 11 & 12,959 & 1 & 13,903 & 12 \\
\hline 10,111 & 5 & 10,973 & 3 & 12,073 & 15 & 12,979 & 60 & 13,963 & 12 \\
\hline 10,133 & 7 & 10,979 & 4 & 12,107 & 4 & 13,001 & 4 & 13,997 & 3 \\
\hline 10,139 & 60 & 10,987 & 9 & 12,119 & 1 & 13,003 & 8 & 13,999 & 5 \\
\hline 10,141 & 8 & 10,993 & 11 & 12,157 & 132 & 13,049 & 4 & 14,033 & 3 \\
\hline 10,151 & 96 & 11,059 & 21 & 12,203 & 1 & 12,063 & 20 & 14,057 & 12 \\
\hline 10,163 & 1 & 11,071 & 113 & 12,241 & 288 & 13,109 & 7 & 14,071 & 69 \\
\hline 10,181 & 24 & 11,083 & 336 & 12,263 & 1 & 13,127 & 108 & 14,081 & 15 \\
\hline 10,211 & 4 & 11,119 & 5 & 12,329 & 207 & 13,163 & 28 & 14,083 & 5 \\
\hline 10,247 & 9 & 11,171 & 1 & 12,347 & 108 & 13,177 & 3 & 14,149 & 11 \\
\hline 10,271 & 1 & 11,173 & 12 & 12,379 & 5 & 13,229 & 15 & 14,159 & 1 \\
\hline 10,289 & 12 & 11,197 & 12 & 12,391 & 8 & 13,241 & 4 & 14,197 & 288 \\
\hline 10,301 & 220 & 11,243 & 57 & 12,401 & 51 & 13,249 & 96 & 14,303 & 1 \\
\hline 10,321 & 3 & 11,273 & 27 & 12,409 & 396 & 13,291 & 5 & 14,387 & 13 \\
\hline 10,331 & 1 & 11,299 & 12 & 12,421 & 3 & 13,313 & 40 & 14,411 & 13 \\
\hline 10,333 & 60 & 11,311 & 65 & 12,437 & 4 & 13,339 & 5 & 14,461 & 20 \\
\hline 10,357 & 3 & 11,317 & 108 & 12,451 & 200 & 13,367 & 4 & 14,537 & 3 \\
\hline 10,429 & 267 & 11,399 & 60 & 12,479 & 4 & 13,397 & 19 & 14,551 & 5 \\
\hline 10,433 & 7 & 11,471 & 1 & 12,527 & 33 & 13,411 & 20 & 14,591 & 25 \\
\hline 10,457 & 3 & 11,497 & 23 & 12,539 & 4 & 13,417 & 12 & 14,593 & 3 \\
\hline 10,513 & 12 & 11,519 & 1 & 12,547 & 129 & 13,451 & 1 & 14,627 & 88 \\
\hline 10,589 & 96 & 11,579 & 1 & 12,553 & 32 & 13,463 & 1 & 14,633 & 40 \\
\hline 10,607 & 13 & 11,617 & 80 & 12,577 & 368 & 13,487 & 4 & 14,639 & 57 \\
\hline 10,613 & 16 & 11,621 & 36 & 12,583 & 8 & 13,537 & 80 & 14,657 & 3 \\
\hline 10,631 & 25 & 11,699 & 1 & 12,611 & 49 & 13,567 & 377 & 14,669 & 4 \\
\hline 10,657 & 104 & 11,701 & 3 & 12,641 & 31 & 13,577 & 3 & 14,699 & 1 \\
\hline 10,663 & 8 & 11,719 & 132 & 12,647 & 340 & 13,619 & 1 & 14,713 & 20 \\
\hline 10,687 & 332 & 11,783 & 1 & 12,671 & 1 & 13,627 & 32 & 14,767 & 5 \\
\hline 10,691 & 1 & 11,813 & 3 & 12,703 & 5 & 13,669 & 11 & 14,779 & 9 \\
\hline 10,733 & 3 & 11,827 & 113 & 12,739 & 45 & 13,679 & 4 & 14,783 & 1 \\
\hline 10,753 & 8 & 11,831 & 1 & 12,757 & 3 & 13,681 & 44 & 14,831 & 1 \\
\hline 10,799 & 1 & 11,833 & 3 & 12,781 & 48 & 13,697 & 3 & 14,843 & 16 \\
\hline 10,837 & 83 & 11,939 & 1 & 12,791 & 1 & 13,751 & 9 & 14,879 & 1 \\
\hline 10,853 & 3 & 11,959 & 5 & 12,823 & 20 & 13,759 & 144 & 14,891 & 16 \\
\hline 10,859 & 25 & 11,969 & 12 & 12,899 & 1 & 13,763 & 1 & 14,939 & 1 \\
\hline 10,861 & 68 & 11,981 & 171 & 12,911 & 21 & 13,799 & 16 & 14,957 & 4 \\
\hline 10,867 & 12 & 12,011 & 1 & & & & & & \\
\hline
\end{tabular}

Received November 28, 1960; revised February 2, 1961. 
10,000 and $q<10,485,760$. The purpose of this note is to present an extension of this table for $10,000<p<15,000$ and $q<10,485,760$.

The table presents the value of $K$ for which $q=2 K p+1$ is the smallest divisor of $M_{p}$ rather than presenting the divisor, $q$. This has been done because, first, the value of $K$ indicates more about the character of the divisor than $q$ does, and, second, to save space. All primes between 10,000 and 15,000 were examined. If any such prime is not listed in the table, it means that $2^{p}-1$ has no prime factor $<10 \cdot 2^{20}$.

Several criteria have been discovered concerning the divisors of the Mersenne numbers, $M_{p}$. The best known of these (for $K=1$ ) is due to Euler [2]. It states that if $p=4 L+3$ and $q=8 L+7$ are both primes then $q$ divides $M_{p}$. For $K=3$, Pellet was the first to state [3] that $q=6 p+1$ divides $M_{p}$ if $q$ can be expressed in the form $4(2 a+1)^{2}+27 b^{2}$, and $p$ and $q$ are both prime. For $K=4$, Reuschle stated and Western proved [4] that $q=8 p+1$ divides $M_{p}$ if $q$ can be expressed in the form $d^{2}+64(2 c+1)^{2}$ and $p$ and $q$ are both prime.

These calculations were performed on an IBM 650 system at Picatinny Arsenal. The program used was as follows: all prime factors $q$ of $M_{p}(p>2)$ are of the form $q=2 K p+1$, and of one of the two forms $8 L \pm 1$. Thus, either $K=0 \bmod$ 4 or $p+K=0 \bmod 4$. Each prime $p$ was expressed in binary form, $p=\sum_{i=0} a_{i} 2^{i}$, $a_{i}=0$ or 1 . The residues $R_{i}=R_{i-1}^{2} \bmod q, R_{0}=2$, were found. Finally the residue $\prod_{i=0}\left(R_{i}\right)^{a_{i}} \bmod q$ was evaluated. If this product is congruent to one $(\bmod q)$ then $q$ is a divisor of $M_{p}$.

\section{Picatinny Arsenal}

Dover, New Jersey

1. H. Rieser, "Mersenne Numbers," MTAC, v. 12, July 1958, p. 207.

2. G. H. HARDY \& E. M. WRIGHT, An Introduction to the Theory of Numbers, 3rd edition, 1956, Oxford University Press, p. 80. 25.

3. L. E. Dickson, History of the Theory of Numbers, v. I, Chelsea Publishing Co., 1952, p.

4. A. E. Western, "Some criteria for the residues of 8 th and other powers, "Proc. London Math. Soc. (2) 9 (1911) p. 244-272.

\section{On a Theorem of Mann on Latin Squares}

\section{By R. T. Ostrowski and K. D. Van Duren}

Mann [1] proved the following theorem: If a Latin square $L$ of order $4 t+2$ has $a(2 t+1) \times(2 t+1)$ block with as many as $(2 t+1)^{2}-t$ cells containing digits in a list of $2 t+1$, then there exists no Latin square orthogonal to $L$. This theorem seemed until lately to give theoretical evidence for the truth of Euler's conjecture that no pair of orthogonal Latin squares exists of any order $4 t+2$. Now that Euler's conjecture has been shown to be false [2], [3], a more detailed investigation of Latin squares of orders $4 t+2$ seems worthwhile.

The chief goal of the work reported in this note was to find an example indicating that Mann's theorem is the best possible of its type for order 10-or conversely, to

Received July 30, 1960. 